\title{
AN EXPERIMENT ON LEARNING APPROPRIATE SELECTIONAL RESTRICTIONS FROM A PARSED CORPUS
}

\author{
Francesc Ribas Framis* \\ Departament de Llenguatges i Sistemes Informàtics, Universitat Politècnica de Catalunya \\ Pau Gargallo 5, 08082 Barcelona, SPAIN. c-mail: ribas@lsi.upc.es
}

\begin{abstract}
We present a methodology to extract Selectional Restrictions at a variable level of abstraction from phrasally analyzed corpora. The method relays in the use of a wide-coverage noun taxonomy and a statistical measure of the co-occurrence of linguistic items. Some experimental results about the performance of the method are provided.
\end{abstract}

Keywords: large text corpora, computational lexicons

\section{INTRODUCTION}

These last years therc has been a common agreement in the natural language processing research community on the importance of having an extensive coverage of the surface lexical semantics of the domain to work with, (specially, typical contexts of use). This knowledge may be expressed at different levels of abstraction depending on the phenomena involved: selectional restrictions (SRs), lexical preferences, col-locations, etc. We are specially interested on SRs, which can be ex. pressed as semantic type constraints that a word sense imposes on the words with which it combines in the process of semantic interpretation. Slis must include information on the syntactic position of the words that are being restricted semantically. For instance, one of the senses of the verb drink restricts its subject to be an animal and its object to be a liquid.

SRs may help a parser to prefer some parses among several grammatical ones [WEB90]. Furthermore, SlRs may help the parser when deciding the sernantic role played by a syntactic complement. Lexicograpliy is also interested in the acquisition of SRs. On the one hand, SRs are an interesting information to be iucluded in dictionaries (defining in context approich). On the other hand, as [CII90] remark, the effort involved in an-

\footnotetext{
"This research has been supported by a grant conceded by the Generalitat de Catalunya, 91-1)OGC-1491. Much of the work reported here was carried out during a visit at the Computer labloratory, University of Cambridge. I am grateful to Ted Brisco: and Horacio Rodriguez by their valuable comments.
}

alyzing and classifying all the linguistic material provided by concordances of use of a word can be extremely labor-intensive. If it was possible to represent, roughly the STis of the word being studied, it could be possible to classify roughly tho concordances autornatically in the different word uses before the lexicographer anialysis.

The possible sources of SRs are: introspection by lexicographers, machine-readable dictionaries, and online corpora. 'The main advantage of the latter is that they provide experimental evidence of words uses. Recontly, several approaches on acquiring different kinds of lexical information from corpora have been developed [BPV92, CGHI191, CH90, Res92]. This paper is interested in cxploring the amenability of using a method for extracting SRs from textual data, in the line of these works. 'I'he airn of the proposed technique is to learn the SRs that a word is imposing, from the analysis of the examples of use of that word contained in the corpus. An illustration of such a learning is shown in Figure 1, where the system, departing from the three eximples of use, and knowing that proseculor, buyer and lawmaker are nouns belonging to the semantic cliss <person, individual > , and that indictment, assurance and legislation are nembers of <legal instrument>, should induce that the verb seek imposes SRs that constraint the subject to be a nember of the semantic type <person, individual>, and the object to be a kind of <legalinstrument>. Concluding, the system should extract for each word (with complementis) having enough number occurrences of use in the corpus and for each of its syntactic complements, a list of the alternative SRs that this word is imposing,

In order to detect the SRs that a word imposes in its context by means of statistical techniques two distiuct approaches have been proposed: word-bused [CGHIT)1], and class.based [BPV92, Res92]. Word. based approach infers SRs as the collection of words that co-occur significantly in the syntactic context of the studied word. The class-based techniques gather the difterent nouns by means of semantic classes. The alvantages of the latter are clear. On the one hand, statistically meaningful data can be gathered from (rel- 
Figure 1: Example of the acquisition of SRs for the verb seek from three examples of use

\section{- Three examples of use}

prosecutors may soon seek an indictment on racketeering and securities fraud charges.

In the recent past, bond buyers didn't seek such assurance.

Some lawmakers may seek legislation to limit overly restrictive insurance policies.

- The extracted SRs

(seek, subject, <person, individual>)

(seek, object, <legal_instrument $>$ )

atively) small corpora, and not only for the most frequent words. On the other hand, SRs are generalized to new examples not present in the training set. $\mathrm{Fi}$ nally, the acquired SRs are more independent of the lexical choices made in the training corpus.

We have developed and implemented a method for automatically extracting class-based SRs from on-line corpora. In section 2 we describe it while discussing other approaches. In section 3 we analyze some data about the performance of an experiment run in a Unix machine, on a corpus of 800,000 words. Finally, in section 4 we discuss the performance achieved, and suggest further refinements of the technique in order to solve some remaining problems.

\section{The Method of Acquisition}

SRs have been used to express semantic constraints holding in different syntactic and functional configurations. However, in this paper we focus only in selectional restrictions holding between verbs and their complements. The method can be easily exported to other configurations. We won't distinguish the SRs imposed by verbs on arguments and adjuncts. We believe that few adjuncts are going to provide enough evidence in the corpus for creating SRs. In the following paragraphs we describe the functional specification of the system.

Training set The input to the learning process is a list of co-occurrence triples codifying the cooccurrence of verbs and complement heads in the corpus: (verb, syntactic relationship, noun). Verb and noun are the lemmas of the inflected forms appearing in text. Syntactic relationship codes the kind of complement: 0 subject, 1 object, or preposition in case it is a PP. A method to draw the co-occurrence triples from corpus is proposed in subsection 2.1 .
Output The result of the learning process is a set of syntactic SRs, (verb, syntactic relationship, semantic class). Semantic classes are represented extensionally as sets of nouns. SRs are only acquired if there are enough cases in the corpus as to gather statistical evidence. As long as distinct uses of the same verb can have different SRs, we permit to extract more than one class for the same syntactic position. Nevertheless, they must be mutually disjoint, i.e. not related by hyperonymy.

Previous knowledge used In the process of learning SRs, the system needs to know how words are clustered in semantic classes, and how semantic classes are hierarchically organized. Ambiguous words must be represented as having different hyperonym classes. In subsection 2.2 we defend the use of a broad-coverage taxonomy.

Learning process The computational process is divided in three stages: (1) Guessing the possible semantic classes, i.e. creation of the space of candidates. In principle, all the hyperonyms (at all levels) of the nouns appearing in the training set are candidates. (2) Evaluation of the appropriateness of the candidates. In order to compare the different candidates, an statistical measure summarizing the relevance of the occurrence of each of the candidate classes is used. (3) Selection of the most appropriate subset of the candidate space to convey the SRs, taking into account that the final classes must be mutually disjoint. While in subsection 2.3 an statistical measure to fulfill stage 2 is prosented, stages 1 and 3 are discussed in 2.4 thoroughly.

\subsection{Extracting Co-occurrence Triples}

In any process of learning from examples the accuracy of the training set is the base for the system to make correct predictions. In our case, where the semantic classes are hypothesized not univoquely from the examples, accuracy becomes fundamental.

Differcnt approaches to obtain lexical co-occurrences have been proposed in the literature [BPV92, CGHH91, CH90]. These approaches seem inappropriate for tackling our needs, either because they detect only local co-occurrences[CGHH91, CH90], or because they extract many spurious co-occurrence triples [BPV92, CH90]. On the one hand, our system intends to learn SRs on any kind of verb's complements. On the other hand, the fact that these approaches extract co-occurrences without reliability on being verbcomplements violates accuracy requirements.

However, if the co-occurrences were extracted from a corpus annotated with structural syntactic information (i.e., part of speech and "skeletal" trees), the results would have considerably higher degrees of accu- 
racy and representativity. In this way, it would be easy to detect all the relationships between verb and complements, and few non-related co-occurrences would be extracted. 'The most serious objection to this approach is that the task of producing syntactic analyzed corpora is very expensive. Nevertheless, lately there has been a growing interest to producc skeletally analyzed corpora $^{1}$

A parser, with some simple heuristics, would be enough to meet the requirements of representaliveness and accuracy introduced above. On the other hand, it could be useful to represent the co-occurrence triples as holding between lemmas, in order to gather as much evidence as possible. A simple morphological analyzer that could get the lemma for a big percentage of the words appearing in the corpus would suffice.

\subsection{Semantic Knowledge Used}

Of the two class-based approaches presented in section 1, [Res92]'s technique uses a wide-coverage scmantic taxonomy, whereas [BPV92] consists in hand-tagging with a fixed set of semantic labels. The advantages and drawbacks of both approaches are diverse. On the one hand, in [BPV92] approach, semantic classes relevant to the domain are chosen, and consequently, the adjustment of the classes to the corpus is quite nice. Nevertheless, [Res92]'s system is less constrained and is able to induce a most appropriate level for the SRs. On the other hand, while [BPV92] implies hand-coding all the relevant words with semantic tags, [Res92] needs a broad semantic taxonomy. However, there is already an available taxonomy, WordNet ${ }^{2}$. We take [Res92] approach because of the better results obtained, and the lower cost involved.

\subsection{Class appropriateness: the Associ- ation Score}

When trying to choose a measure of the appropriateness of a semantic class, we have to consider the features of the problem: (1) robustness in front of noise, and (2) conservatism in order to be able to gencralize only from positive examples, without having the tendency to over-generalize.

Several statistical measures that, accomplish these requirements have been proposed in the literature [BPV92, CGHH191, Res92]. We adopt [Res92]'s approach, which quantifies the statistical association

\footnotetext{
'For instance, Penn Treebank Corpus, which is being collected and analyzed by the University of Pennsylvania (sec [MSM93]). The material is available on request fion the [inguistic Data Consortium, (email) ldc(3unagi.cis.npenu.cdu

${ }^{2}$ WordNet is a lexical database developed with psycholit guistic aims. It represents lexical semanties information about nouns, verbs, adjectives and adverbs such as hyperonyms, meronyms, ... It presently contains information on abont 83,000 lemmas. See $[\mathrm{MBF}+90]$
}

between verbs and classes of nouns from their cooccurrence. However we arlapt it taking into account the syntactic position of the relationship. Let

$$
\begin{gathered}
V=\left\{v_{1}, \ldots, v_{l}\right\}, \mathcal{N}=\left\{n_{1}, \ldots, n_{m}\right\}, \\
S=\{0,1, t o, a t, \ldots\}, \text { and } \mathcal{C}=\{c \mid c \subseteq \mathcal{N}\}
\end{gathered}
$$

be the sets of all verbs, nouns, syntactic positions, and possible noun classes, respectively. Given $v \in \mathcal{V}$, $s \in \mathcal{S}$ and $c \in \mathcal{C}$, Association Score, Assoc, between $v$ and $c$ in a syrtactic position $s$ is defined to be

$$
\begin{gathered}
A \operatorname{ssoc}(v, s, c) \equiv P(c / v, s) I(v ; c / s) \\
=P(c / v, s) \log _{2} \frac{P(v, c / s)}{P(v / s) P(c / s)}
\end{gathered}
$$

Where conditional probabilities are estimated by counting the number of observations of the joint event and dividing by the frequency of the given event, e.g

$$
P(v, c / s) \approx \frac{\sum_{n \in \mathcal{C}} \operatorname{count}(v, s, n)}{\sum_{v^{\prime} \in \mathcal{V}} \sum_{n^{\prime} \in \mathcal{N}} \operatorname{count}\left(v^{\prime}, s, n^{\prime}\right)}
$$

The two terms of $A$ ssoc try to capture different properties of the SR expressed by the candidate class. Mutual information, $I(v ; c / s)$, measures the strength of the statistical association between the given verb $v$ and the candidate class $c$ in the given syntactic po sition $s$. If there is a real relationship, then hopefully $I(v, c / s) \gg 0$. On the other hand, the conditional probability, $P(c / v, s)$, favors those classes that have more occurrences of nouns.

\subsection{Selecting the best classes}

The existence of noise in the training set introduces classes in the candidate space that can't be considered as expressing SRs. A common technique used for ignoring as far as possible this noise is to consider only those events that have a higher number of occurrences than a certain threshold. Ilowever, some erroncous classes may persist because they exceed the threshold. How ever, if candidate classes were ordered by the significance of their Assoc with the verb, it is likely that less appropriate classes (introduced by noise) would be ranked in the last positions of the candidate list.

The algorithm to learn SRs is based in a search through all the classes with more instances in the training set than the given threshold. In different iterations over these candidate classes, two operations are performed: first, the class, $c$, having the best $A \operatorname{ssoc}$ (best class), is extracted for the final result; and second, the remaining candidate classes are filtered from classes being hyper/hyponyms to the best class. This last step is made because the definitive classes must be mutually disjoint. The iterations are repeated until the candiditte space has been run out. 
Table 1: SRs acquired for the subject of seek

\begin{tabular}{|c|c|c|c|c|c|}
\hline Acquired $S R$ & Type & Assoc & $\# n$ & $\# s$ & Examples of nouns in Treebank \\
\hline$\langle$ cognition $\rangle$ & Senses & -0.04 & 5 & 1 & concern, leadership, provision, science \\
\hline$\langle$ activity $\rangle$ & Senscs & -0.01 & 6 & 1 & administration, leadership, provision \\
\hline$<$ status $>$ & Senses & 0.087 & 5 & 0 & government, leadership \\
\hline$<$ social_control $>$ & Senses & 0.11 & 6 & 0 & administration, government \\
\hline$\left.<a d m i n i s t r a t i v e \_d i s t r i c t\right\rangle$ & Senses & 0.14 & 36 & 0 & proper_name \\
\hline$<$ city $\rangle$ & Senses & 0.15 & 36 & 0 & proper_name \\
\hline$<$ radical $>$ & Senses & 0.16 & 5 & 0 & group \\
\hline$\langle$ person, individual $\rangle$ & Ok & 0.23 & 61 & 38 & advocate, buyer, carrier, client, company, ... \\
\hline$<$ legal_action $>$ & Ok & 0.28 & 7 & 6 & suit \\
\hline$<$ group $>$ & $\Uparrow A b s$. & 0.35 & 64 & 46 & administration, agency, bank, ..., group, ... \\
\hline$\langle$ suit $\rangle$ & Sensès & 0.40 & 7 & 0 & suit \\
\hline$<$ suit_of_clothes $>$ & Senses & 0.41 & 7 & 0 & suit \\
\hline$<$ suit, suing $\rangle$ & Senses & 0.41 & 7 & 0 & suit \\
\hline
\end{tabular}

[Res92] performed a similar learning process, but while he was only looking for the preferred class of object nouns, we are interested in all the possible classes (SRs). He performed a best-first search on the candidate space. However, if the function to maximize doesn't have a monotone bchavior (as it is the case of Assoc) the best-first search doesn't guarantee global optimals, but only local ones. This fact made us to decide for a global search, specially because the candidate space is not so big.

\section{Experimental Results}

In order to experiment the methodology presented, we implemented a system in a Unix machine. The corpus used for extracting co-occurrence triples is a fragment of parsed material from the Penn Trecbank Corpus (about 880,000 words and 35,000 sentences), consisting of articles of the Wall Street Journal, that has been tagged and parsed. We used Wordnet as the verb and noun lexicons for the lemmatizer, and also as the semantic taxonomy for clustering nouns in semantic classes. In this section we evaluate the performance of the methodology implemented: (1.) looking at the performance of the techniques used for extracting triples, (2) considering the coverage of the WordNet taxonomy regarding the noun senses appearing in Treebank, and (3) analyzing the performance of the learning process.

The total number of co-occurrence triples extracted amounts to 190,766 . Many of these triples $(68,800$, $36.1 \%$ ) were discarded before the lommatizing process because the surface NP head wasn't a noun. The remaining 121,966 triples were processed through the lemmatizer. $113,583(93.1 \%)$ could be correctly mapped into their corresponding lemma form.
In addition, we analyzed manually the results obtained for a subset of the extracted triples, looking at the sentences in the corpus where they occurred. The subset contains 2,658 examples of four average common verbs in the Treebank: rise, report, seek and present (from now on, the testing sample). On the one hand, $235(8.8 \%)$ of these triples were considered to be extracted erroneously because of the parser, and 51 $(1.9 \%)$ because of the lemmatizer. Summarizing, 2,372 $(89.2 \%)$ of the triples in the testing set were considered to be correctly cxtracted and lemmatized.

When analyzing the coverage of WordNet taxono$\mathrm{my}^{3}$ we considered two different ratios. On the one hand, how many of the noun occurrences have one or more senses included in the taxonomy: 113,583 of the 117,215 extracted triples $(96.9 \%)$. On the other hand, how many of the noun occurrences in the testing sample have the correct sense introduced in the taxonomy: $2,615$ of the 2372 well-extracted triples ( $8.7 \%)$. These figures give a positive evaluation of the coverage of WordNet.

In order to evaluate the performance of the learning process we inspected manually the SRs acquired on the testing-sample, assessing if they corresponded to the actual SRs imposed. A first way of evaluation is by means of measuring precision and recall ratios in the testing sample. In our case, we define precision as the proportion of triples appearing in syntactic positions with acquired SRs, which effectively fulfill one of those SRs. Precision amounts to $79.2 \%$. The remaining $20.8 \%$ triples didn't belong to any of the classes induced for their syntactic positions. Some of them because they didn't have the correct sense included in the WordNet taxonomy, and others because the correct class had not been induced because there wasn't

\footnotetext{
${ }^{3}$ The information of proper nouns in WordNet is poor. For this reason we assign four predefined classes to them $\langle$ person, individtal $\rangle$, $\langle a d m i n i s t r a b i v e d i s t r i c t\rangle$ and $\langle$ city $\rangle$.
} 
enough evidence. On the other hand, we define recall as the proportion of triples which fulfill one of the SRs acquired for their corresponding syntactic positions. Recall amounts to $75.7 \%$.

A second way of evaluating the performance of the abstraction process is to manually diagnose the reasons that have made the system to deduce the SRs obtained. Table 1 shows the SRs corresponding to the subject position of the verb seek. Type indicates the diagnostic about the class appropriateness. $A s s o c$, the value of the association score. "\# $n$ ", the number of nouns appearing in the corpus that are contained in the class. Finally, "\# $s$ " indicates the number of actual noun senses used in the corpus which are contained in the class. In this table we can see some examples of the five types of manual diagnostic:

Ok The acquired SR is correct according to the noun senses contained in the corpus.

$\Uparrow A$ bs The best level for stating the SR is not the one induced, but a lower one. It happens because erroneous senses, metonymies, ..., accumulate evidence for the higher class.

$\Downarrow$ Abs Some of the SRs conld be best gathered in a unique class. We didn't find any such case.

Senses 'The class has cropped up bccause it accumulates enough evidence, provided by erroneous senses.

Noise The class accumulates enough evidence provided by erroneously extracted triples.

Table 2 shows the incidence of the diagnostic types in the testing sample. liach row shows: the type of diagnostic, the number and percentage of classes that accomplish it, and the number and percentage of noun occurrences contained by these classes in the testing sample 4. Analyzing the results obtained from the testing sample (some of which are shown in tables 1 and 2) we draw some positive $(a, e)$ and some negative conclusions $(b, c, d$ and $f$ ):

a. Almost one correct semantic class for each syntactic position in the sample is acquired. 'I'he technique achieves a good coverage, even with few co. occurrence triples.

b. Although many of the classes acquired result from the accumulation of incorrect senses $(73.3 \%)$, it seems that their size tends to be smaller than classes in other categories, as they only contiin a $51,4 \%$ of the senses.

\footnotetext{
"this total doesn't equal the number of triples in the testing sample because the same noun may belong to nore than one class in the final SRs
}

Table 2: Summary of the SRs acquired

\begin{tabular}{|c|c|c|c|c|}
\hline Diagnostic & $\#$ Classes & $\%$ & $\# n$ & $\%$ \\
\hline Ok & 45 & 18.8 & 2,099 & 39.4 \\
\hline$\Uparrow \Lambda \mathrm{bs}$ & 7 & 2.9 & 362 & 6.8 \\
\hline$\Downarrow \wedge \mathrm{bs}$ & 0 & 0.0 & 0 & 0.0 \\
\hline Senses & 176 & 73.3 & 2,740 & 51.4 \\
\hline Noíse & 12 & 5.0 & 130 & 2.4 \\
\hline Total & 240 & 100.0 & 5,331 & 100.0 \\
\hline
\end{tabular}

c. 'There doesn't seem to be a clear co-relation between Assoc and the manual diagnostic. Specifically, the classes considered to be correct sometimes aren't ranked in the higher positions of the Assoc (e.g., Table 1).

d. The over-gencralization seems to be produced because of little difference in the nouns included in the rival classes. Nevertheless this situation is rarc.

e. The impact of noise provided by erroneous extraction of co-occurrence triples, in the acquisition of wrong semantic classes, seems to be very moderatc.

f. Since different verb senses occur in the corpus, the SRs acyuired appear mixed.

\section{FURTHER WORK}

Although perfornance of the technique presented is protty good, some of the detected problems could possibly be solved. Specifically, there are various ways to explore in order to reduce the problems stated in points $b$ and $c$ above:

1. To measure the $\Lambda$ ssoc by means of Mutual Information between the pair $v-s$ and $c$. In this way, the syntactic position also would provide information (statistical evidence) for measuring the most appropriate classes.

2. To modify the Assoc in such a way that it was based in a likelihood ratio test [Dun93]. It seems that this kind of tests have a better performance than mutual information when the counts are small, as it is the case.

3. To estimate the probabilities of classes, not directly from the frequencies of their noun mem. bers, but correcting this evidence by the number of senses of those nouns, e.g

$$
I^{\prime}(c / s) \approx \frac{\sum_{n \in C} \operatorname{count}(v, s, n) \frac{\# \operatorname{senses}(n) \in \mathcal{C}}{\# \operatorname{senses}(n)}}{\sum_{v^{\prime} \in V} \sum_{n^{\prime} \in \mathcal{N}} \operatorname{count}\left(v^{\prime}, s, n^{\prime}\right)}
$$


In this way, the estimated function would be a probability distribution, and more interesting, nouns would provide evidence on the occurrence of their hyperonyms, inversely proportional to their degree of ambiguity.

4. To collect a bigger number of examples for each verbal complement, projecting the complements in the internal arguments, using diathesis subcategorization rules. Hopefully, Assoc would have a better performance if it was estimated on a bigger population. On the other hand, in this way it would be possible to detect the SRs holding on internal arguments.

In order to solve point $d$ above, we have foreseen two possibilities:

1. To take into consideration the statistical significance of the alternatives involved, before doing a generalization step, climbing upwards,

2. To use the PPs that in the corpus are attached to other complements and not to the main verb as a source of "implicit negative examples", in such a way that they would constrain the overgeneralization.

Finally, It would be interesting to investigate the solution to point $f$. One possible way would be to disambiguate the senses of the verbs appering in the corpus, using the SRs already acquired and gathering evidence of the patterns corresponding to each sense by means of a technique similar to that used by [Yar92]. Therefore, once disambiguated the verb senses it would be possible to split the set of SRs acquired.

\section{References}

[BPV92] R. Basili, M.T. Pazienza, and P. Velardi. Computational lexicons: the neat examples and the odd exemplars. In Proc. of the $3 r d$ ANLP, 1992.

[CGHH91] K.W. Church, W. Gale, P. Hanks, and D. Hindle. Using statisties in lexical analysis. In U. Zernik, editor, Lexical Acquisition: Exploiting On-Line Resources to Build a Lexicon. Lawrence Erlbaum, 1991.

[CH90] K.W. Church and P. Hanks. Word association norms, mutual information and lexicography. Computational Linguistics, 16(1), 1990 .

[Dun93] T. Dunning. Accurate methods for the statistics of surprise and coincidence. Computational Linguistics, 19(1), 1993.
$\left[\mathrm{MBF}^{+} 90\right]$ G. Miller, R. Beckwith, C. Fellbaum, D. Gross, and K. Miller. Five papers on wordnet. Technical report, CSL, Princeton University, 1990.

[MSM93] Mitchell P. Marcus, Beatrice Santorini, and Mary Ann Marcinkiewicz. Building a large annotated corpus of english: the Penn Treebank. Computational Linguistics, 19(2), 1993.

[Res92] P. Resnik. Wordnet and distributional analysis: A class-based approach to lexical discovery. In Proc. of $A A A I$ Workshop on Statistical Methods in NLP, 1992.

[WFB90] G. Whittemore, K. Ferrara, and H. Brunnex. Empirical study of predictive powers of simple attachment schemes for postmodifier prepositional phrases. In Proc. of the 28th ACL, 1990.

[Yar92] David Yarowsky. Word-sense disambiguation using statistical models of Roget's categories trained on large corpora. In Proceedings of COIING-92, Nantes, France, 1992. 